\title{
APPLICATIONS OF GAS CHROMATOGRAPHY TO ANAESTHESIOLOGY 1. GASES AND VAPOURS*
}

Howard L. Zauder, M.D., PH.D., ANd Louis R. Orkin, Mr.d.

The Quantitative Determination of anaesthetic vapours contained in a gas mixture has, until recently, usually been accomplished by chemical analysis. The classical procedures, of which the Orsat and its modifications ${ }^{1}$ are perhaps the most representative, consist of metering a definite volume of a gas mixture into a burette, bringing the gas into contact with a reagent which will react with or absorb the particular constituent being determined, and finally measuring the change in volume resulting from removal of the constituent vapour. This procedure is then repeated with different reagents for successive removal of each of the components of the mixture until the entire composition is determined. Analysis by this technique is quite obviously slow and tedious. In addition, several common errors are possible. These include those due to transfer of samples, gas leaks in the apparatus, and incomplete removal of the constituent of the mixture by the appropriate reagent. Alternatively, one may employ a "conversion technique." This involves conversion of the particular constituent to a non-volatile by-product. which is then determined quantitatively by some physical or chemical method. The conversion technique most commonly employed for analysis of the anaesthetic agents utilized the iodine pentoxide train in its original or modified form.? Here a sample containing a gas or vapour is drawn through a tube filled with asbestos, which in turn is impregnated with iodine pentoxide. The organic substances are oxidized to carbon dioxide and water by the iodine pentoxide. In the process, free iodine or hydrogen iodide or both are liberated. These substances are then quantitatively titrated with a sodium bisulphite or thiosulphate solution. The disadvantages of this method combine those of the Orsat technique with the disadvantages of organic analysis and finally of quantitative titration of the by-products.

Because of the obvious disadvantages of the chemical methods of analysis. many investigators have, in recent years, turned to physical means of assay. These methods measure some physical property of a gas or mixture of gases and thereby give information as to the quantitative composition. Physical methods may be divided into two groups: specific and non-specific. The non-specific methods, which include analysis of density, ${ }^{3}$ thermal conductivity, ${ }^{4}$ refractive index, ${ }^{5}$ the velocity of sound through gases, ${ }^{6}$ and paramagnetism, ${ }^{7}$ measure a property which is possessed to varying degrees by all gases. The specific methods, including absorption of radiant energy in the infra-red ${ }^{8}$ and invisible ${ }^{\theta}$ regions of the spectra,

\footnotetext{
*From the Department of Anesthesiology, Albert Einstein College of Medicine, New York 61, N.Y. Supported in part by Contract No. DA 49-007-MD 962, Research and Development Command, Office of the Surgeon General, United States Army, and United States Public Health Service Grant No. 2762.
}

Can. Anaes. Soc. J., vol. 10, no. 3, May, 1963 
gas chromatography, ${ }^{10}$ and polarography, ${ }^{11}$ measure a property possessed only by the gas being analysed. The relative advantages and disadvantages of the physical methods have recently been reviewed by Mapleson. ${ }^{12}$

Anaesthesiologists, and others concerned with quantitation of gas and vapour mixtures, have long sought an analytical technique that would permit rapid and accurate quantitative analysis of these compounds. Ideally, the technique should not only be quantitative, but should permit qualitative separation of the agents from each other as well as from the fixed respiratory gases. It would be advantageous if the method was universal, i.e., applicable for all vapours and gases that are or may be employed in anaesthesia, and if it could eliminate costly and time-consuming physical and chemical methods of analysis.

Gas chromatography appears to satisfy all these criteria. This technique, introduced approximately a decade ago, has found wide industrial application. ${ }^{13}$ Its potential as a tool in medical research has been recognized only recently. ${ }^{14}$ The first mention of its use in anaesthesiology was by Fabian and Cannes in 1960.10 It has since been used by Hall ${ }^{15}$ for qualitative separation of the halothane-ether azeotrope, and by Glover and Hodgson ${ }^{16}$ for determination of the purity of a series of fluorinated hydrocarbons of anaesthetic interest. Several other investigators have used this technique as a tool in the quantitation of inspired atmospheres of anaesthetic gases. ${ }^{17}$ There is some indication that gas chromatography will be applicable to the determination of anaesthetic levels in blood and other body fluids. ${ }^{4} 14: 20$ The basic principles of gas chromatography were reviewed by Summers and Adriani. ${ }^{21}$

In adapting the technique to the quantitative analysis of anaesthetic drugs, we found several unanswered questions. How accurate is the method? How reproducible are the results? What temperatures and carrier gas flows should be employed for a particular anaesthetic? Which column should be employed for any particular agent? In this and the subsequent reports, we shall attempt to answer these questions in the hope that others will be saved considerable time in putting this technique into routine use.

\section{Principles of Instrunentation}

Basically, gas chromatography is an adsorption technique utilizing a physical method of separation in which the components to be separated are distributed between two phases. One phase consists of a stationary bed of large surface area while the other phase is a gas or vapour that percolates through or along the stationary bed. As with other types of chromatography, resolution of a mixture is based on the affinity of the compounds for the stationary phase. ${ }^{22}$ Two types of stationary beds were employed. A solid phase in the form of a molecular sieve was used for quantitation of rare gases of anaesthetic interest: the commonly employed anaesthetic agents were separated on a stationary phase consisting of an organic liquid of high boiling point coated on inert granules (diatomaceous earth). The gases or vapours being quantitated are swept into the column by the streaming of an inert carrier gas. Those gases which are strongly attracted 
to the stationary phase move through the column at a slow rate and appear at the end of the column later than the components which have demonstrated an indifference towards the stationary bed. Substances are thus concentrated by the column and eluded in a pure form. The rate of adsorption and elution, e.g., retention time, is dependent upon the temperature of the column, flow-rate of the carrier gas through the column, and finally upon the nature of the packing or active material of the column.

When a surge of gas appears at the end of a column, it may be detected in several ways. Detection by thermal conductivity was employed in the analysis of the gases and vapours of anaesthetic interest. Detectors of this type (thermistors) are constructed of ceramic materials of a high thermal coefficient. Small changes in the composition of the gas surrounding the detectors alter the thermal conductivity of the mixture. Small changes in thermal conductivity markedly alter the electrical resistance of the thermistor. This alteration in electrical resistance is measured by upsetting the balance on a Wheatstone bridge which incorporates the reference and sensing detector in opposing limbs. The unbalance is amplified and recorded on a potentiometric recorder. As each component of a mixture is detected a characteristic curve or chromatogram is produced. Eluded substances can be readily identified by comparison with a known or predetermined chromatogram. In addition, the area of the inscribed curve is related to the concentration of the substance passing through the detector chamber. The apparatus is depicted schematically in Figure 1. It should be noted that in addition to a pres-

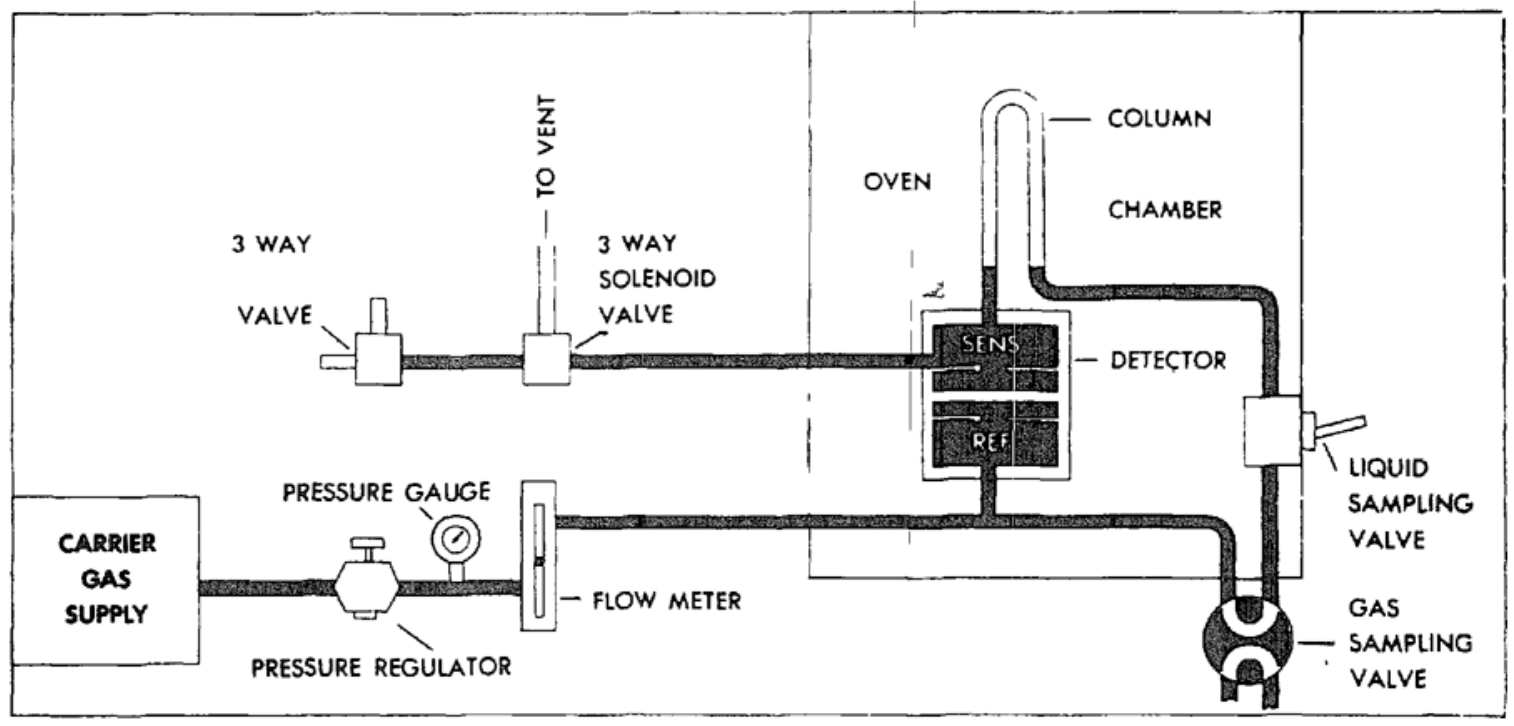

FIGURE 1. Block diagram of the chromatograph. Provision is made for regulation of carrier gas flow, maintenance of constant temperature of the column and detector block, and constant volume sampling.

sure regulator, pressure gauge, and flow-meter to regulate carrier gas supply, the chamber is thermostatically controlled so that the temperature of the column and detectors is maintained within $\pm 0.1^{\circ} \mathrm{C}$. The apparatus is also provided with a constant-volume sampling valve for gases and with an injector block for liquid samples. Helium is most commonly employed as the carrier gas. 


\section{Apparatus}

There are several highly satisfactory gas chromatographs available commercially. Alternatively, one may, if he is particularly ambitious or mechanically gifted, fabricate his own apparatus. ${ }^{23}$ The instrument used in our laboratory was a Perkin-Elmer 154-C Vapor Fractometer. The bridge circuit across the sensing and reference thermistors was coupled to a Leeds-Northrup Type G Speedomax Strip Chart Recorder. The five-millivolt full-scale response time of this recorder was one second. Signal output from the bridge could be attenuated by factors of two down to $1 / 512$ of maximum sensitivity. This permits adjustment of peakheight signals from the detector block. A valley sensor and transmitting potentiometer of a Perkin-Elmer Model 194 Printing. Integrator was attached to the recorder pen drive shaft. The areas of the peaks produced by the eluded gases as they flowed through the thermal conductivity cell were thus automatically integrated and recorded. The current across the bridge circuit was kept constant at 8 volts.

\section{Operating Conditions}

The rapidity with which a sample of gas can traverse the column is dependent upon three factors: the flow-rate of the carrier gas through the column, the nature of the column itself, and finally the temperature of the column. In this investigation, each of these factors was varied independently until the optimum combination was determined. The effect of increasing flow-rate of carrier gas upon retention time using a $1 / 4$ in., 2-metre DC 200 silicone oil (dimethyl siloxane polymer) column and holding the temperature constant is shown in Figure 2. Obviously retention time can be reduced by increasing the rate of flow of carrier gas. A flow

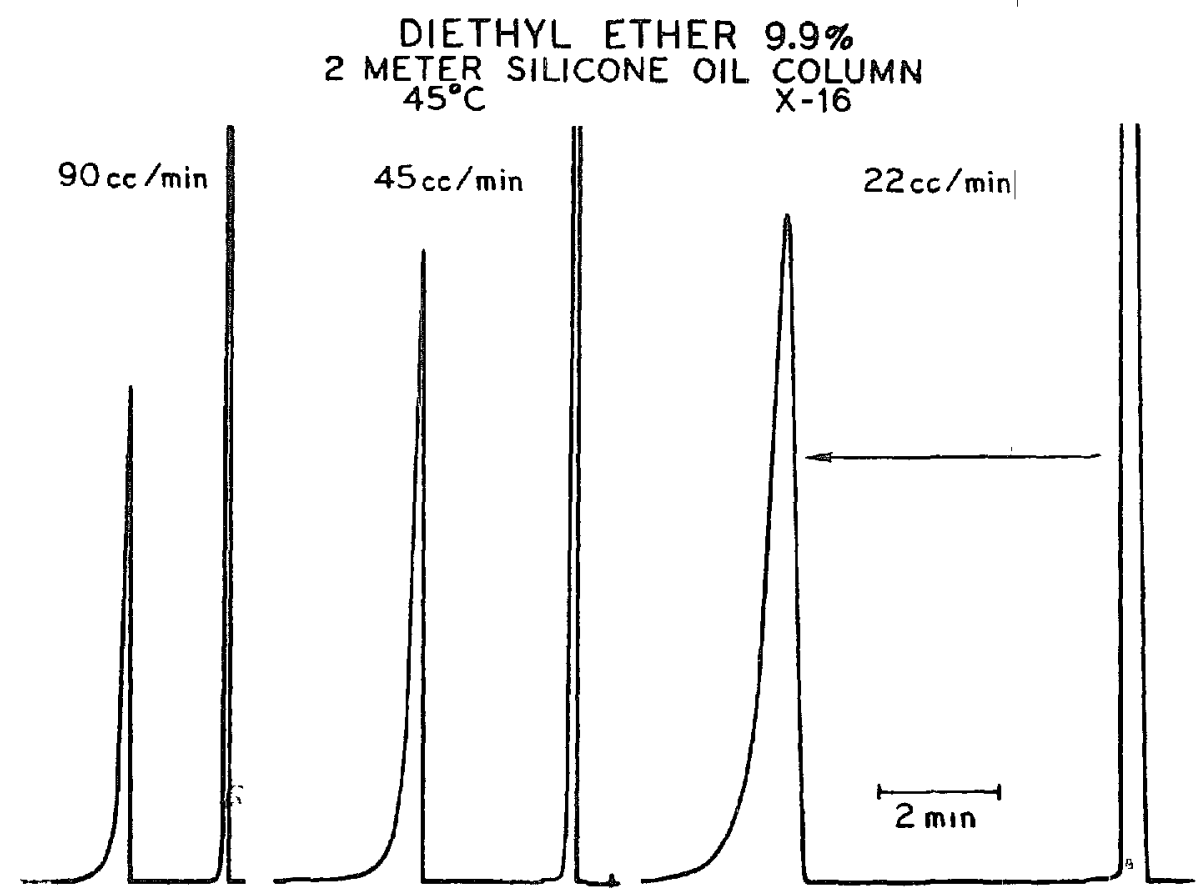

Figure 2. Decrease in retention time of diethyl ether when flow-rate of carrier gas is increased. The initial peak represents room air. 


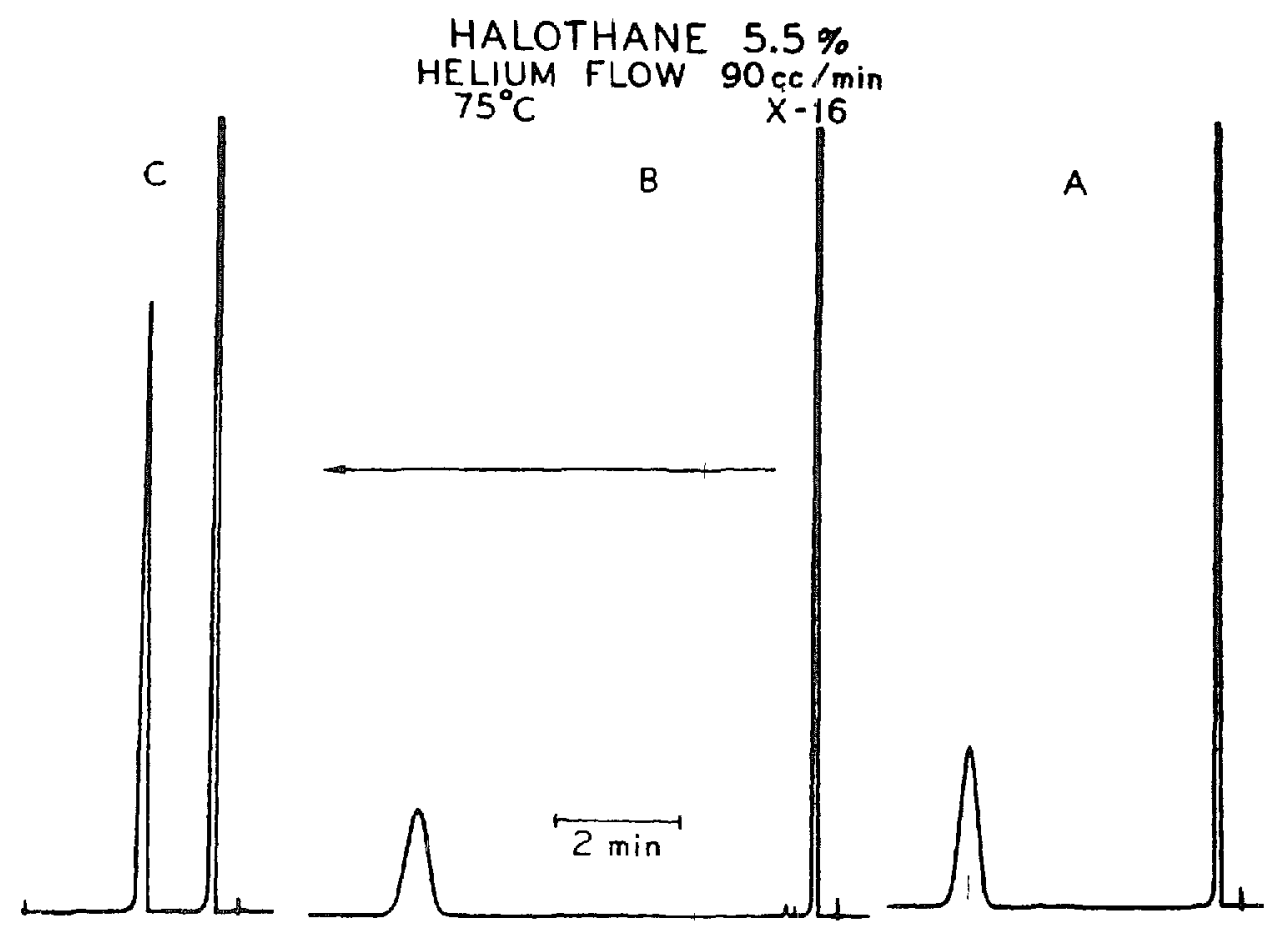

Figure 3. Effect of column packing on retention time. $\mathrm{A}=$ di-isodecylphthalate, $\mathrm{B}=$ di-2-ethylhexyl sebacate, $\mathrm{C}=$ dimethyl siloxane polymer ( $\mathrm{DC}$ 200 silicone oil).

of 90 c.c. $/ \mathrm{min}$. was found to be at the optimum for anaesthetic agents. At higher flow-rates there is incomplete separation of the components. Fusion of the peaks made rapid identification and quantitation difficult. At lower flow-rates read-out time was needlessly prolonged. The effect of varying the column packing with all other factors being held constant is shown in Figure 3. Retention time is shortest with a silicone oil (C) column and longest with a di-2-ethylhexyl sebacate column (B). The di-isodecylphthalate column (A) occupies an intermediate position. In each instance the column was 11 in. in diameter and 2 metres in length. When a 1-metre column was employed, separation was often incomplete. The effect of temperature upon retention time with the three differently packed columns is shown in Figure 4. The speed with which a given substance is eluded from the column is inversely proportional to the temperature of the column. Except for nitrous oxide, retention time is lowest on a silicone oil column (C). It should be noted that a column temperature at least $10^{\circ} \mathrm{C}$. above ambient is necessary to give stability to the system. It was seldom necessary to operate the system above $75^{\circ} \mathrm{C}$.; liquid anaesthetic agents of high boiling point could be eluded at this temperature in less than five minutes. The higher the temperature employed, the shorter was the useful life-span of the column. At high temperatures the organic liquid of the stationary phase boils off, requiring periodic renewal of the column. For routine analysis a temperature $\left(75^{\circ} \mathrm{C}\right.$.) that was consistent with rapid determination of the compounds in question was employed.

Although any of the three columns can be used, the silicone oil column was chosen for rapid analysis at a temperature consistent with a long column life. It is recognized that a "tailing-off" effect is seen when divinyl or diethyl ether 

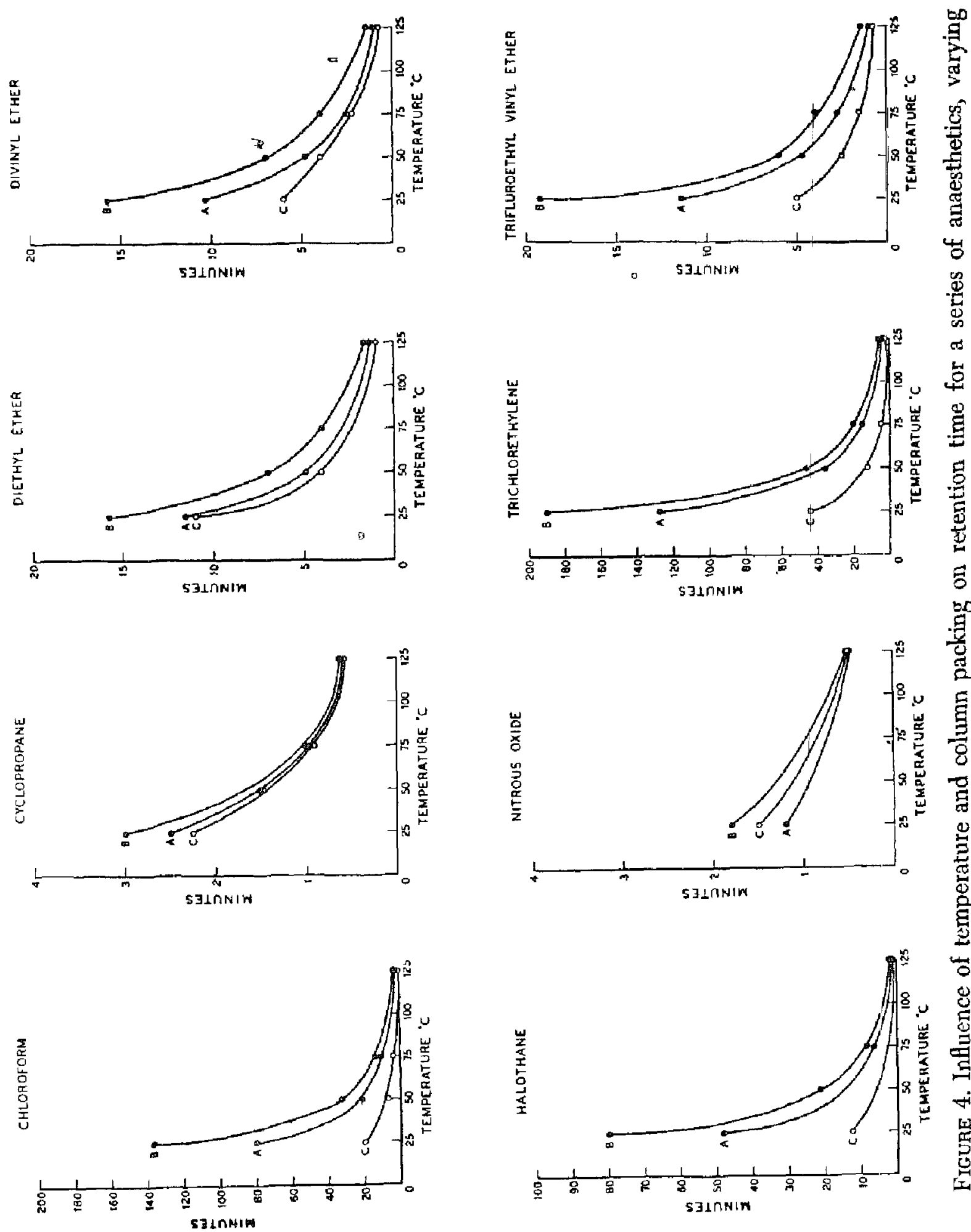

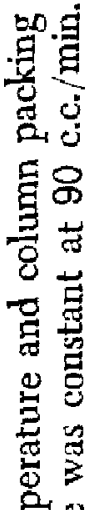

实㐘

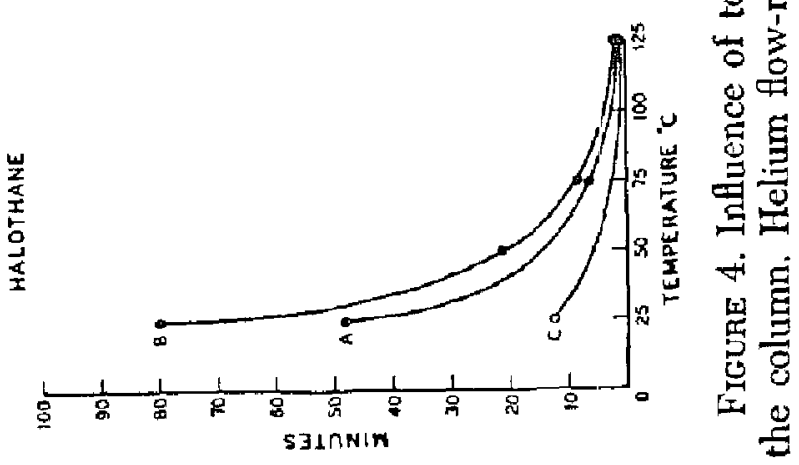


(Figure 2) is quantitated on this column. The practical accuracy of the results is not affected by the asymmetry of the curve.

\section{Preparation of Standards}

Standard gas mixtures were made in gas burettes. Following preparation they were transferred to gas-tight bottles. Standards of volatile agents were made by vapourizing a known amount of liquid anaesthetic, delivered from a calibrated syringe, in a bottle of known volume. The gas mixture was agitated constantly by a magnetic spin bar in the bottle. Volumes were corrected for the space occupied by the bar. Aliquots for standardization of the apparatus were drawn into 50-c.c. syringes previously rendered air-tight by spraying with teflon aerosol. It was tound that the preparation of the standard was the greatest potential source of error in the entire procedure. With some practice, however; it was possible to prepare standards with a great deal of reproducibility.

\section{Calculation of Results}

The chromatogram (i.e., the curve inscribed by the recorder when the detector bridge is unbalanced) may be related to the concentration of gas or vapour passing through the detector cell in one of two ways. The first entails determination of the area under the curve. This area, in turn, may be found in several ways: (1) by electronic integration, (2) gravimetrically, (3) by planimetry, (4) by mathematical integration, or (5) it may reasonably be approximated by

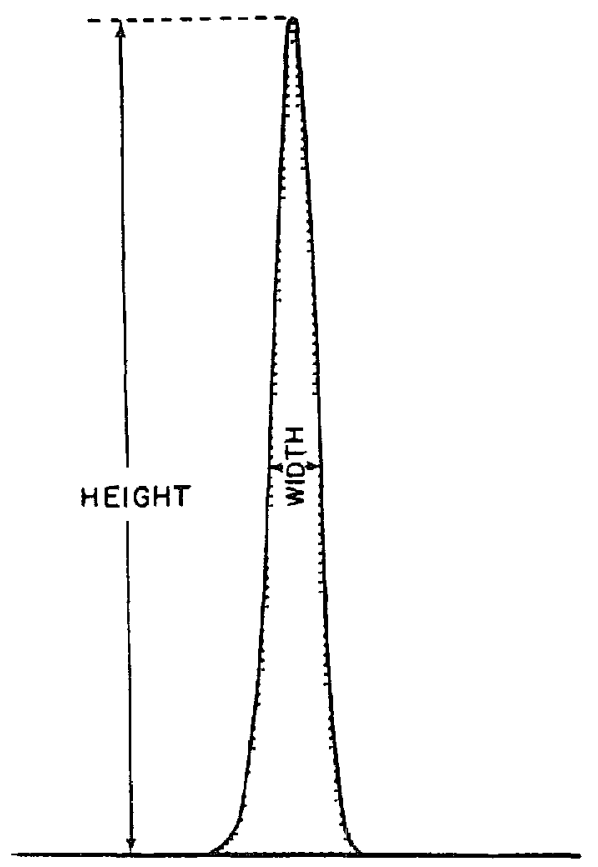

Figure 5. The chromatogram can be related to concentration by determining the area under the curve (stippled) or by measuring the peak height. In some instances the area can be approximated by calculating height times width at onehalf the height. 
multiplying the height of the peak by the width of the peak at one-half the height (Fig. 5). Another method of handling the data is to relate the peak height of the chromatogram to the concentration of the gas or vapour. This, the simplest and least expensive way of handling the data, is quite accurate in the case of these peaks (Table I)

The relation of the area to the percentage concentration of eight commonly employed anaesthetic agents is shown in Figure 6. The relationship is linear over the entire range examined. In each case a 2-metre silicone oil column was employed; operating conditions for the individual anaesthetics are given. The plots are the averages of three determinations of at least six standards. The fit of the curve to each of these points is a reflection of the high degree of accuracy obtainable with this method. Those points which lie somewhat off the plotted line
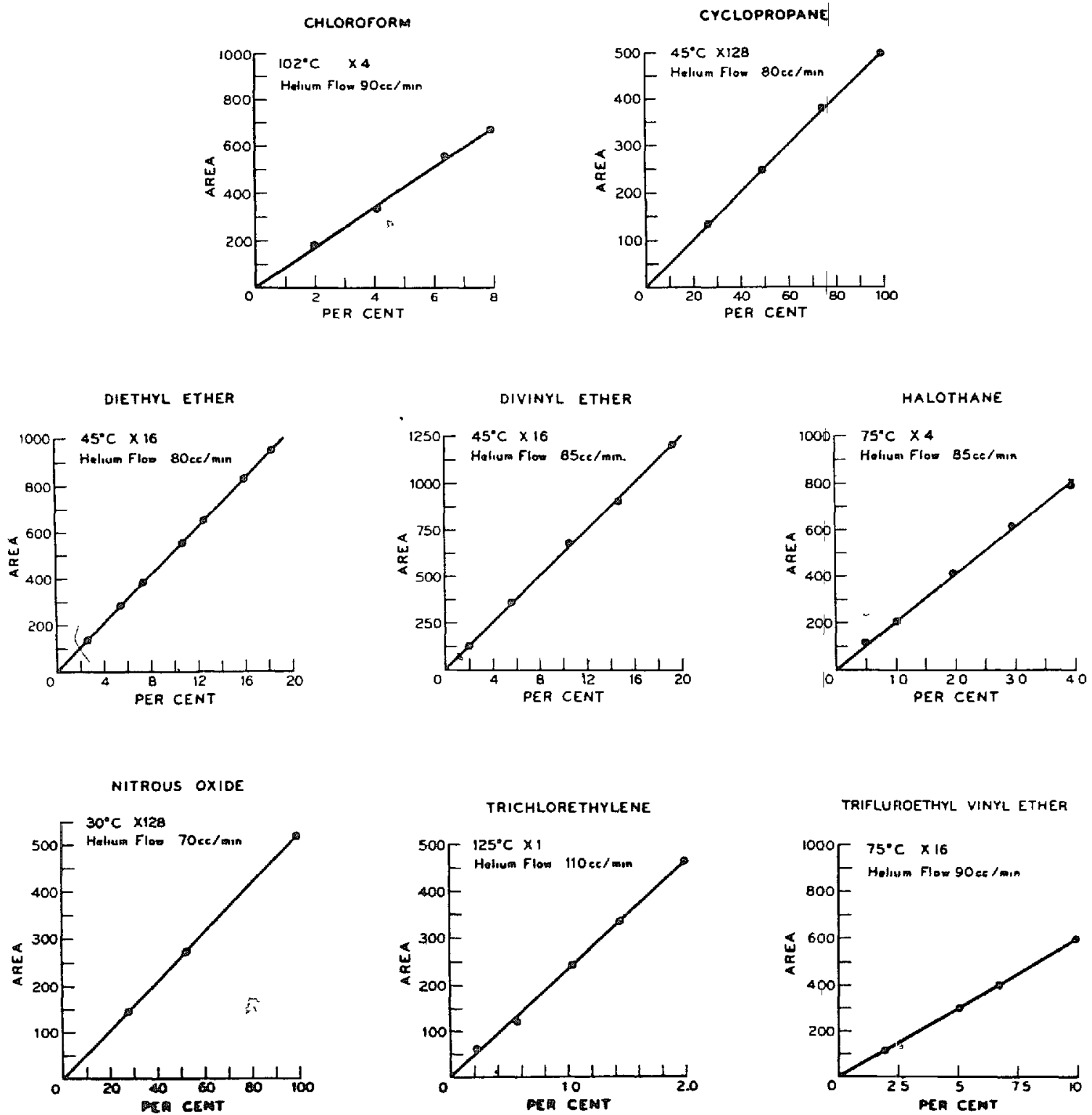

Figure 6. Relation of area to concentration utilizing a 2-metre silicone oil column. Temperatures, flow-rate, and attenuation are given for each anaesthetic agent. 
TABLE I

Concentration Calculated by Electronic Integration, Height, and Approximate Area*

\begin{tabular}{lcccc}
\hline \hline & Concentration, \% & Area ${ }^{*} \dagger$ & Height ${ }^{*} \dagger$ & $\begin{array}{c}\text { Approximate } \\
\text { Area } \$\end{array}$ \\
\hline Diethyl ether & 6.8 & \pm 0.04 & \pm 0.05 & \pm 2.7 \\
Divmyl ether & 5.3 & \pm 0.03 & \pm 0.03 & \pm 2.2 \\
Halothane & 2.0 & \pm 0.01 & \pm 0.01 & \pm 0.8 \\
Cyclopropane & 20.0 & \pm 0.09 & \pm 0.08 & \pm 4.4 \\
Trichloroethylene & 2.4 & \pm 0.02 & \pm 0.01 & \pm 0.7 \\
Chloroform & 4.0 & \pm 0.03 & \pm 0.04 & \pm 2.0 \\
\hline
\end{tabular}

"Standard deviation, 20 samples each.

$\$$ By electronic integration.

率 Measured in $\mathrm{cm}$.

\$Determined from measurement of peak height and width at one-half the peak height.

are less likely to represent inaccuracy of the analytical method than an inability to prepare accurately standard gas and vapour mixtures.

The linearity and accuracy as reflected by these curves greatly simplify the method of analysis in that only one standard is needed to construct the calibration curve for any particular agent. Using a known concentration that is approximately in the range of the unknowns and by adjusting the attenuation so that this concentration gives approximately full-scale deflection on the recorder it is possible to increase the resolution of the analytical method to a point not ordinarily achieved by other methods of physical or chemical analysis with any degree of ease. The concentration of an unknown sample can be determined from a calibration curve or since the concentration is directly proportional to the peak area or peak height, an unknown can be determined from the following equation:

where $C_{1}=$ concentration of known,

$$
C_{2}=C_{1} A_{1} / A_{2}
$$

$C_{2}=$ concentration of unknown,

$A_{1}=$ area or height of known,

$A_{2}=$ area or height of unknown.

\section{RePRoducibility of Results}

Twenty runs of the same concentration were successively made to determine the reproducibility of the method. These results are summarized in Table I. It will be observed that the degree of reproducibility for twenty samples is virtually the same when analysis of the chromatogram is made by integration of the area under the curve and by measurements of peak heights. When one approximates the area under the curve by multiplying height of the peak by the width at onehalf the height, the method becomes quite unreliable. This is, no doubt, a reflection of inability to measure, with sufficient accuracy, the width of these narrow peaks. For this reason this method of interpretation of the data was abandoned early. 


\section{Other ApPLICATIONS}

Separation of mixtures of several anaesthetic agents is possible utilizing this technique. This includes resolution of the fluo-ether azeotrope, which is not separable, by other physical or chemical methods (Fig. 7). When this figure is

FLUO -E THER

2 METER SILICONE OIL COLUMN

HELIUM FLOW $90 \mathrm{cc} / \mathrm{min}$

$30^{\circ} \mathrm{C} \times 16$

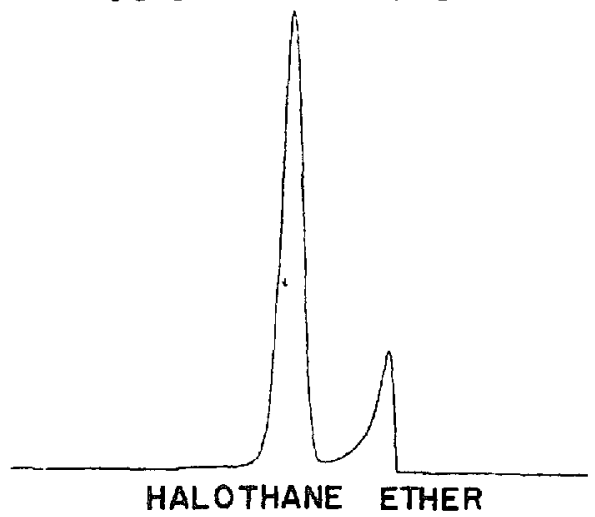

Figure 7. Resolution of fluo-ether. "Tailing-off" of the ether peak is well demonstrated.

\section{METER SILICONE OIL COLUMN $100^{\circ} \mathrm{C} \times 16$ \\ HELIUM FLOW $90 \mathrm{cc} / \mathrm{min}$}

Frgure 8. Chromatograms of methoxyfluorane and of a fluorinated hydrocarbon of potential use as an anaesthetic. 
compared with the chromatograms obtained by $\mid \mathrm{Hall}_{,}^{1.5}$ it is noted that the total time for separation and elution is considerably shorter and that the peaks are somewhat sharper. This is referable to the difference in operating conditions, that is, temperature, flow-rate of carrier gas, and nature of the column packing. With the columns in current use it has not been possible to separate a mixture of diethyl and divinyl ether.

The applicability of this method for the determination of new compounds of anaesthetic interest is shown in Figure 8. These chromatograms represent 4.8 per cent methoxyfluorane and 3.2 per cent methyl-1,1-difluro-2-bromofluro-ethyl ether respectively. In addition to quantitating the amounts of vapour contained in a mixture it is also possible, utilizing this technique, to determine the presence of impurities in the mixtures. Figure 9 is a chromatogram of a gaseous fluorinated hydrocarbon of potential anaesthetic interest that contains a contaminant.

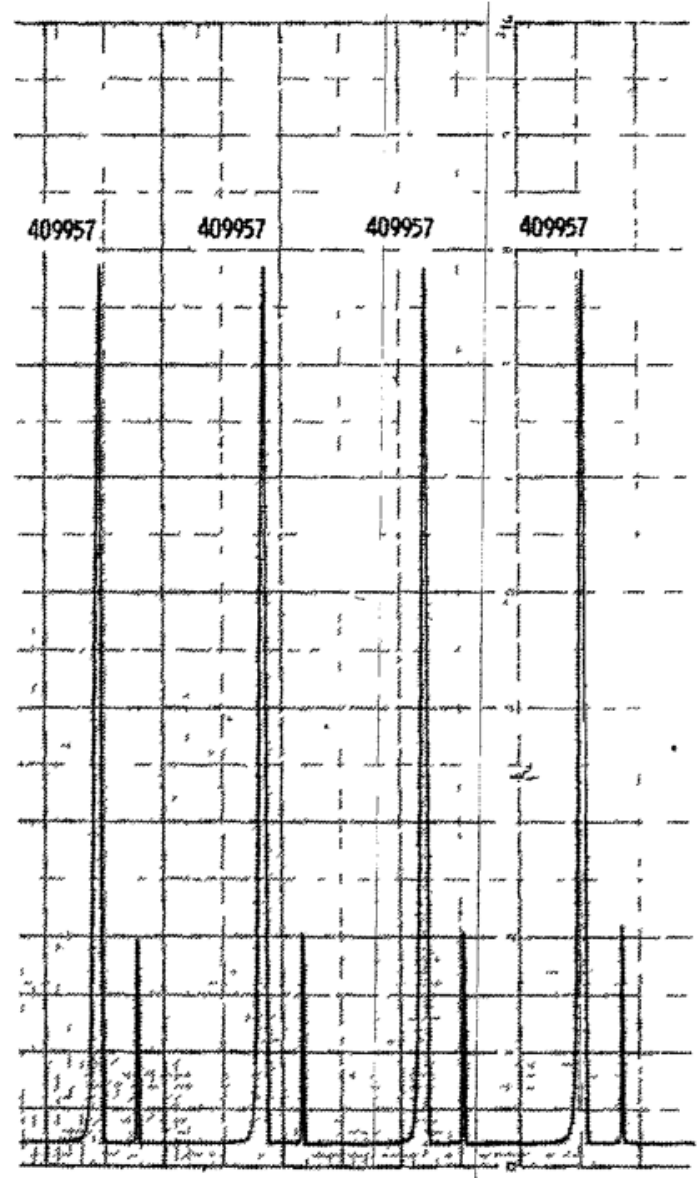

FIGURE 9. Presence of a constant contammant (short peaks) in a gaseous fluorinated hydrocarbon being investigated for anaesthetic properties.

It has been possible, utilizing gas-solid chromatography, to resolve and analyse quantitatively a mixture of the rare gases that exhibit anaesthetic properties. Employing a 2-metre ${ }_{4}^{1 /}$-in. molecular sieve column, it was possible to resolve a mixture of 20 per cent oxygen and 80 per cent xenon (Fig. 10). Similarly one can resolve mixtures of oxygen and krypton. In the case of xenon, the time 


\section{METER MOLECULAR SIEVE COLUMN \\ HELIUM FLOW $60 \mathrm{cc} / \mathrm{m}$ in \\ $70^{\circ} \mathrm{C}$ \\ $x-16$}

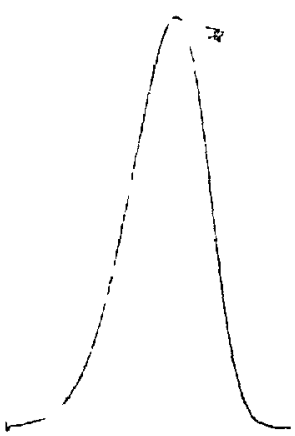

$\mathrm{Xe}$

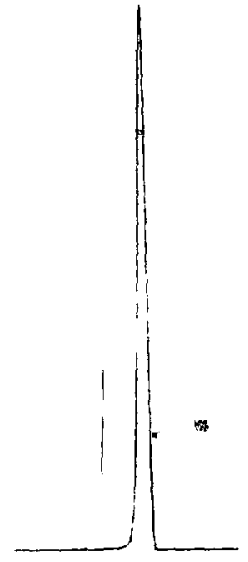

$\mathrm{O}_{2}$

Figure 10. Resolution of an oxygen-xenon mixture.

required for separation was 18 minutes. This should be hastened somewhat by elevating the column temperature.

\section{Conclusion}

Analysis of anaesthetic gases and vapours by gas chromatography is accurate, simple, and fast. Most of the agents possess thermal conductivities of sufficient magnitude so that a small change in the composition of a mixture produces sufficient change in the chromatogram to allow accurate analysis. There can be no doubt that physical methods of analysis are more accurate and less tedious than the time-honoured chemical procedures. The majority of the physical analytical techniques require expensive, complicated, fickle instrumentation, which in turn must be operated by trained, skilled technicians. Gas chromatography, utilizing a thermal conductivity detector, while hardly inexpensive, is a relatively simple technique which can be mastered by the average technician in a matter of hours. As is the case in all analytical techniques, the accuracy and reproducibility of the determination is dependent upon the accuracy with which standard gas mixtures can be prepared. This is the limiting factor in the effective use of this technique. It should be emphasized that in spite of statements to the contrary, this is essentially a research tool and at present has no place in the monitoring of the routine clinical anaesthetic.

The application of this technique, in anaesthesiology, is currently limited to analysis of volatile and gaseous agents, and fixed respiratory gases. The horizons, however, are unlimited. Barbiturates, phenothiazines, narcotics, amino acids, and amines of biological interest can all be quantitated by slight modifications of the chromatographic technique described. 


\section{RÉSUMÉ}

Pour faire l'analyse d'une série de gaz et de vapeurs anesthésiques, nous avons employé la chromatographie des gaz en nous servant d'un détecteur à conduction thermique. Ce système ne coûte pas cher, est facile à calibrer et à faire fonctionner; il permet une répétition des analyses et donne une précision qu'on ne peut obtenir que difficilement avec les autres méthodes physiques ou chimiques d'analyse. On peut employer ce système pour l'analyse de tous les agents anesthésiques d'usage courant, et nous l'avons employé pour mesurer plusieurs nouveaux produits d'un intérêt possible pour les anesthésiologistes. Nous présentons une étude détaillée des facteurs pouvant influencer la vitesse de l'analyse.

\section{REFERENCES}

1. Fieluner, A. G.; Jones, G. W; \& Holbrook, W. F. The Bureau of Mines Orsat Apparatus for Gas Analysis. Bureau of Mines Technical Paper No. 320 (1925).

2. SHEPHER, M. An Improved Apparatus and Method for the Analysis of Gas Mixtures by Combustion and Absorption. Bur. Standards J. Research Paper No. 266 (1931),

3. Waller, A. D. The Chloroform Balance. J. Physiol. 37: VI (1908).

4. Roвbins, B. H. Cyclopropane: A Method for Quantating Cyclopropane in Air and Blood: Concentrations of Cyclopropane in the Air and Blood Necessary for Anesthesia, Loss of Reflexes and Respiratory Arrest. J. Pharmacal. \& Exper. Therap. 58: 243 (1936).

5. Hall, K. D.; Garimgtion, L. H.; Nowirl, W. K.; \& Ste.phen, C. R. The Analysis of Small Concentrations of Trichlorethylene Vapor by Interferometry. Anesthesiology 14. 38 (1953).

6. Molyneux, L. \& PAsk, E. A. A Sonic Analyser for Anaesthetic Vapors, Anaesthesia 14: 191 (1959).

7. Pauling, L.; Wood, R. E.; \& Sturdivant, J. M. An Instrument for Determining Partial Pressure of Oxygen in a Gas. Science 103: 338 (1946).

8. Colliter, C. R.; Affelnt, J. E.; \& Farr, A. F. Continuous Rapid Infra-red Carbon Dioxide Analysis. J. Lab. \& Clin. Med. 45: 526 ( 1955).

9. Jones, C. S.; Saari, J. M.; Devlow, R. A.; Faulconer, A.; \& Blades, E. J. Analysis of Gases in Blood with a Mass Spectrometer. Anesthesiology 14:490 (1953).

10. Fabian, L. W. \& Carnes, M. A. Gas Chromatography as an Analytical Tool in Anesthesiology. Anesthesiology 21: 98 (1960).

11. Bracken, A.; Glover, J. H.; \& Longmutr, I. S. A New Method of Oxygen Determination. Lancet 2: 411 (1960).

12. Mapleson, W. W. Physical Methods of Gas Analysis. Brit. J. Anaesth. 34: 631 (1962).

13. Cremer, E. \& Muller, R. Separation of Substances by Chromatography. Mikrochim. Acta 36: 533 (1951).

14. Burchfield, H. P. \& Storns, E. E. Biochemical Applications of Gas Chromatography. New York: Academic Press (1962).

15. Hall, K. D.; Norris, F.; \& Downs, S. Physical Chemistry of Halothane-Ether Mixtures. Anesthesiology 21: 522 (1960).

16. Glover, J. H. \& Hodgson, H. W. An Investigation of New Fluorine Compounds in Anaesthesia: Analysis by Gas Chromatography. Anaesthesia 16: 19 (1961).

17. Mapelson, W. W. The Rate of Uptake of Halothane Vapour in Man. Brit. J. Anaesth. 34: 11 (1962).

18. Noehren, T. H. \& Codmone, J. W. Ethyl Ether Content in Blood as Determined by Gas Chromatography. Anesthesiology 22: 519 (1961).

19. Galla, S. J. \& Ottenstein, D. M. Measurement of Inert Gases in Blood by Gas Chromatography. Ann. N.Y. Acad. Sci. 102: 4 (1962).

20. Gadsden, R. H.; MCCond, W. M.; Woods, E. F.; \& Bagwell, E. E. Gas Chromatographic Determination of Methoxyflurane in Blood. Anesthesiology 23: 831 (1962).

21. Summers, F. W. \& Adriani, J. Gas Chromatography: An Analytical Method for Anesthesiology Research. Anesthesiology 22: 100 (1961).

22. Keulemans, A. I. M. Gas Chromatography (edited by C. G. Verver). New York: Reinhold (1959).

23. Butler, R. A. Gas Chromatography as a Method for Estimating Concentrations of Volatile Anaesthetics in Blood. Brit. J. Anaesth. 34: 440 (1962). 